\title{
Cortical Ribbon Sign in Acute Hepatic Encephalopathy with Sequential Atrophy
}

\author{
Ryan T. Muir (i), Arun N. E. Sundaram
}

Keywords: Magnetic resonance imaging, Diffusion restriction, Prion disorder, Creutzfeldt-Jakob disease, Encephalopathy

doi:10.1017/cjn.2019.285

Can J Neurol Sci. 2019; 46: 758-759

A 59-year-old man presented with confusion, decreased level of consciousness, and generalized tonic-clonic seizures. He was intubated and promptly stabilized on antiepileptic medications. He was not in status epilepticus. He improved after seizure control, though he remained confused. He was neither acutely intoxicated nor were there any substance withdrawal concerns prior to his presentation. Furthermore, no metabolic, electrolyte, or nutritional perturbations were identified. He did, however, have a history of alcoholic hepatitis and was awaiting a liver transplant, but his blood work did not reveal evidence of fulminant hepatic failure at presentation (international normalized ratio - 1.17 , platelet count $161,000 / \mu \mathrm{L}$, ammonia $18 \mu \mathrm{mol} / \mathrm{L}$, blood urea nitrogen $4.5 \mathrm{mmol} / \mathrm{L}$, and his liver enzymes were only remarkable for an elevated alkaline phosphatase of $143 \mathrm{U} / \mathrm{L}$ ).

Magnetic resonance imaging (MRI) brain at 13 days after presentation (Figure 1) revealed progression of cortical diffusion restriction in bilateral frontal lobes, the left superior parietal lobe, bilateral insular cortices, and left temporal lobe compared to an initial MRI brain performed at 7 days after presentation. There was also diffusion restriction in bilateral caudate nuclei. This radiographic progression was noted despite clinical and neurophysiologic evidence of seizure control.

Cortical ribbon sign is typical of prion disorders ${ }^{1}$ but can be seen in cortical infarction, status epilepticus, infection, hypoxia, metabolic, electrolyte, and mitochondrial disorders. $^{2,3}$ It has also been reported in acute hepatic encephalopathy. ${ }^{4}$ In the absence of other metabolic or electrolyte derangements, objective evidence of infection, or intoxication, our leading differential diagnoses included hepatic encephalopathy and a prion disease. While elevated plasma ammonia level has been correlated with diffusion restriction in hepatic encephalopathy, not all cases of hepatic encephalopathy with cortical diffusion restriction will have elevated ammonia. ${ }^{4}$ To note, our patient did not have an elevated ammonia level. The pattern of subcortical and cortical diffusion restriction in combination with fluid attenuated inversion recovery imaging has previously been suggested to be highly sensitive and specific for sporadic Creutzfeldt-Jakob disease (CJD). ${ }^{1,2}$ However, the absence of cognitive or behavioral symptoms prior to his presentation was less supportive of a diagnosis of CJD.

Cerebrospinal fluid analysis was entirely unremarkable, and 14-3-3 protein was negative. Serial MRIs ultimately demonstrated resolution of diffusion restriction with evolving bilateral frontal lobe atrophy and encephalomalacia over the course of 7 months (Figure 2). The patient gradually recovered, but he developed frontal lobe dysfunction characterized by new impulsive behaviors.

In conclusion, while the classic differential diagnosis for cortical and subcortical diffusion restriction includes prion disorders such as CJD, this imaging pattern does have a broad differential diagnosis. Even in the context of normal liver function tests and ammonia level, acute hepatic encephalopathy can still produce this radiographic pattern. Furthermore, while cortical diffusion restriction has been described to be reversible, this case highlights the neuroimaging sequelae of cortical diffusion restriction. ${ }^{4}$ Serial MRIs of our case showcase an infrequently depicted phenomenon in the literature to date - the development of encephalomalacia and atrophy, occurring months after a recovery from an episode of acute hepatic encephalopathy.

\section{Disclosures}

RTM and ANES do not have any conflicts of interest to disclose.

\section{Statement of Authorship}

RTM: study conception and design and manuscript preparation. ANES: study conception and design, manuscript preparation, acquisition of data, and final approval of manuscript.

\section{REFERENCES}

1. Vitali P, MacCagnano E, Caverzasi E, et al. Diffusion-weighted MRI hyperintensity patterns differentiate CJD from other rapid dementias. Neurology. 2011;00(20):1711-19. doi:10.1212/ WNL.0b013e31821a4439

2. Rosenbloom MH, Tartaglia MC, Forner SA, et al. Metabolic disorders with clinical and radiologic features of sporadic

From the Division of Neurology, Department of Medicine, University of Toronto, Toronto, Ontario, Canada (RTM, ANES); Department of Ophthalmology and Vision Sciences, University of Toronto, Toronto, Ontario, Canada (ANES).

Received August 6, 2019. Date of Acceptance August 19, 2019.

Correspondence to: Arun N. E. Sundaram, Sunnybrook Health Sciences Centre, Department of Ophthalmology and Vision Sciences, University of Toronto, M1202C, M wing, First Floor, 2075 Bayview Ave., Toronto, Ontario M4N 3M5, Canada. Email: Arun.Sundaram@sunnybrook.ca 
Creutzfeldt-Jakob disease. Neurol Clin Pract. 2015;5(2):108-15. doi:10.1212/CPJ.0000000000000114

3. Sheerin F, Pretorius PM, Briley D, Meagher T. Differential diagnosis of restricted diffusion confined to the cerebral cortex. Clin Radiol. 2008;63(11):1245-53. doi:10.1016/j.crad.2007.12.018
4. McKinney AM, Lohman BD, Sarikaya B, et al. Acute hepatic encephalopathy: diffusion-weighted and fluid-attenuated inversion recovery findings, and correlation with plasma ammonia level and clinical outcome. Am J Neuroradiol. 2010;31(8): 1471-79. doi:10.3174/ajnr.A2112
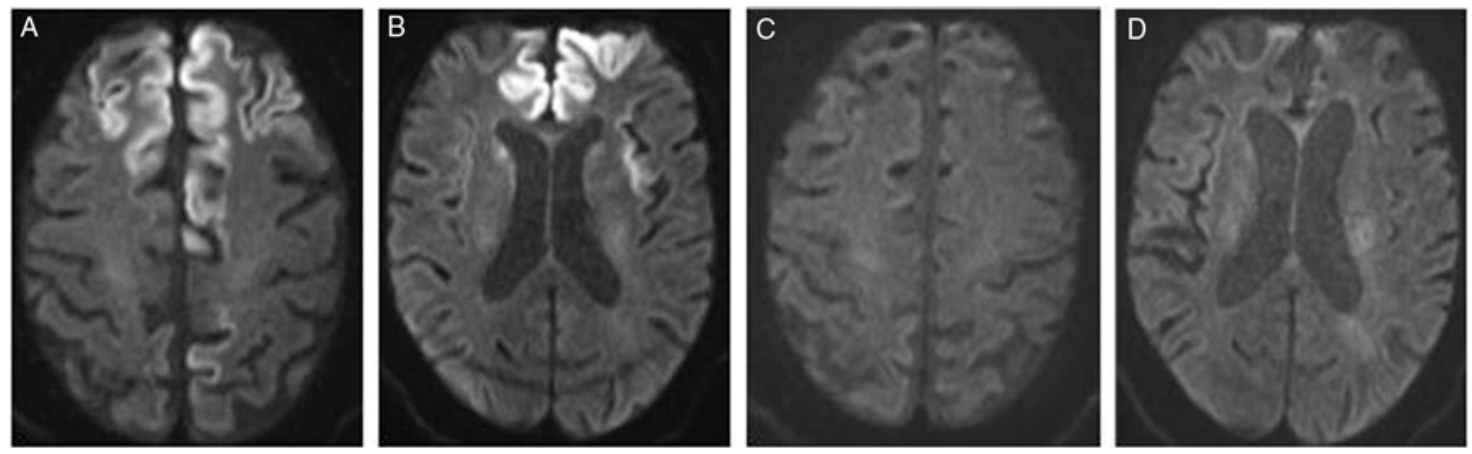

Figure 1: Diffusion weighted MRI at (A) 13 days and (B) 7 months after presentation, demonstrating resolution of cortical diffusion restriction.
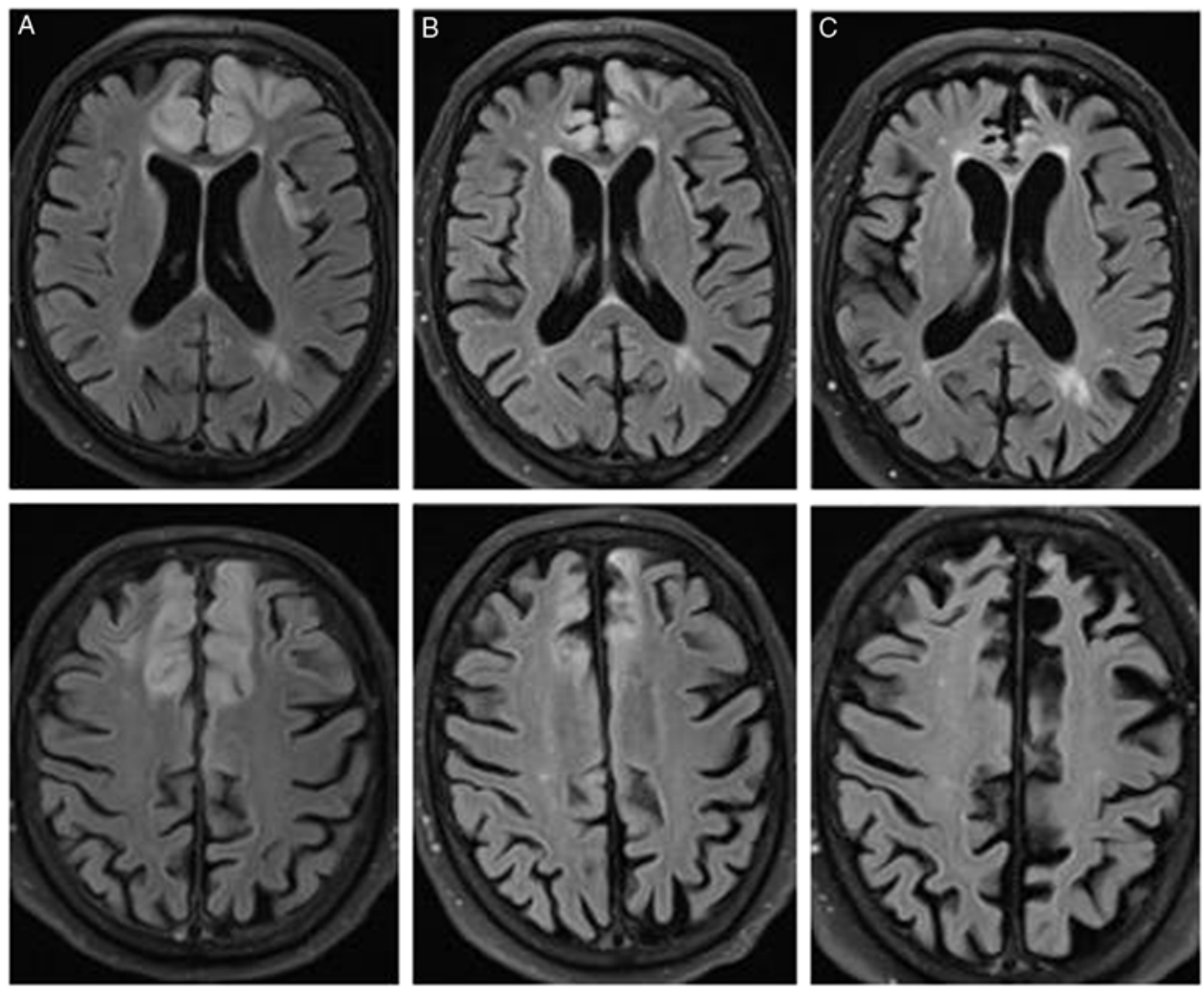

Figure 2: T2-FLAIR MRI at (A) 13 days, (B) 32 days, and (C) 7 months after presentation. This demonstrates resolution of $T 2$ signal change and evolution of frontal atrophy with encephalomalacia. 